\title{
Non-Rigid Image Registration using a Modified Fuzzy Feature-based Inference System for 3D Cardiac Motion Estimation
}

\author{
Monire Sheikh Hosseini ${ }^{\mathrm{a}}$, Mahammad Hassan Moradi ${ }^{\mathrm{a}(*)}$, Mahdi Tabassian ${ }^{\mathrm{b}}$, Jan D'hooge $^{\mathrm{b}}$ \\ a. Department of biomedical engineering, Amirkabir University of Technology, Tehran, Iran \\ ${ }^{\mathrm{b}}$ Laboratory on Cardiovascular Imaging and Dynamics, Department of Cardiovascular Sciences, KU \\ Leuven, Belgium
}

\begin{abstract}
Background and Objective: Non-rigid image registration is a well-established method for estimating cardiac motion on 3D echocardiographic images. However, such images have relatively poor spatio-temporal resolution making registration a challenging task. Some of the main challenges are extracting features relevant to the registration problem and defining a suitable geometrical transformation to be applied to the features. The latter can be tackled using a fuzzy inference system considering its potential in transformation modeling. From this point of view, feature-based image registration can be considered as an identification problem in which the transformation parameters are computed through an optimization problem. This paper, thus, aims to estimate cardiac motion on 3D echocardiographic images based on feature-based non-rigid image registration through sets of modified fuzzy rules. Methods: The 3D volume features are extracted with the popular SIFT (ScaleInvariant Feature Transform) descriptor in 3D space. Sets of fuzzy rules are generated according to the extracted features to register every two consecutive frames. Finally, some supplementary rules modify the registration rule for estimating cardiac motion. Results: Results of applying the fuzzy feature-based inference system on the STRAUS synthetic database are comparable with those of other well-established registration algorithms in terms of tracking error and accuracy of strain estimates. The proposed algorithm had an acceptable performance with a tracking error of $1 \mathrm{~mm}$ and a relative circumferential strain error of $0.82 \pm 4.69 \%$. In addition, applying the algorithm on in-vivo database made it feasible for clinical application. Conclusion: This study indicates the proposed method can track complex cardiac deformations in 3D echocardiographic images.
\end{abstract}

Keywords-SIFT, fuzzy inference system, non-rigid registration, motion estimation, 3D echocardiography

\section{INTRODUCTION}

$\mathrm{T}$ HREE-dimensional (3D) echocardiography has become a well-established imaging technique for cardiac function assessment in daily clinical practice given that it is a noninvasive, relatively cheap, and portable modality that can be used bedside. Despite these favorable characteristics, the resulted 4D data (i.e., 3D images acquired over time) have

${ }^{*}$ Corresponding author: Department of Biomedical Engineering, Amirkabir University of Technology, Tehran, Iran. E-mail addresses: sheikhosseini.m@aut.ac.ir (M. Sheikhhosseini), mhmoradi@aut.ac.ir (M.H. Moradi). relatively poor spatio-temporal resolution which challenges an algorithm designed for quantification of myocardial deformation. Moreover, the quality of the 3D transthoracic echocardiographic (TTE) images can further be reduced due to speckle noise [1] and image artifacts [2]. This makes cardiac motion estimation based on the 3D TTE images a challenging task and introduces uncertainty in the estimates.

Modeling this uncertainty, thus, seems reasonable during the process of motion estimation in order to reliably quantify functional characteristics of the heart. A suitable framework for performing this task is 'fuzzy theory' which can handle both intrinsic and extrinsic uncertainties in such a process and has a wide range of applications in different image processing fields including feature extraction [3]-[5], image thresholding [6], image segmentation [7], curve alignment [8], [9], motion estimation [10] and point-set matching [11]-[14]. Considering the potential of the fuzzy theory and the uncertainties (i.e., fuzziness) associated with the 3D TTE cardiac motion estimation, a fuzzy non-rigid registration algorithm is proposed for efficient quantification of cardiac function.

\subsection{Related works}

Several approaches have been proposed for fuzzy image registration. In some studies, the registration task was considered a regression problem that can be solved through the fuzzy theory [15]. In [16], the fuzzy concept was used to regularize the deformation field while in [17], the authors exploited the fuzzy concept to aid the tracking algorithm. Some other studies made use of fuzzy logic in the different phases of image registration such as segmentation, feature extraction, and similarity matching [4], [18]-[23]. However, only a few studies focus on the ability of the fuzzy systems to model the complex non-rigid deformation of the heart during the cardiac cycle as an image registration task.

Intensity-based and feature-based methods are two main categories for non-rigid registration [24]. Efficient performance of the latter method depends on a set of features that properly 
describe the image characteristics. When feature-based methods have extracted such a feature set, the task of the image registration algorithm is to assign correspondence between two feature sets and recover a geometrical transformation between related point sets (i.e. the position of extracted features). Quantification of the nonlinear relations between the point sets can be seen as system modeling or a system identification problem which can be solved appropriately through the Fuzzy Inference System (FIS) [25]. One of the most straightforward FIS techniques is Takagi-Sugeno-Kang (TSK), which has widely been used for system modeling [26]-[28]. A plethora of studies developed consequent functions in the TSK model for different purposes. For instance, Kalhor et al. used high order TSK to identify the complicated processes like a steam generator model [29]. In [30], the TSK model was transferred to a piecewise affine model. Given the nonlinear yet complex motion of the myocardium, a non-rigid registration algorithm was used in this study to estimate the cardiac motion and clinical parameters.

\subsection{Contributions}

The novelty of the current study lies in formulating a nonrigid registration problem as a fuzzy feature-based inference system. More specifically, the contributions of this work are as follows: 1) describing spatial properties of the 3D TTE images based on the scale-invariant feature transform (SIFT) [31] descriptor, 2) generating fuzzy rules for registering two images based on the features extracted by SIFT and, 3) estimating the cardiac motion by the modified generated rules.

The rationale for using SIFT was twofold. First, the SIFT algorithm is well-known to be resistant against common image deformations [32]. Second, the SIFT features encode valuable regional information of an image which makes this feature extraction approach a favorable choice for the image registration problem [7], [20], [33]. Given that complicated deformations of the myocardium are caused by different regional transformations, local registration algorithms have been shown to be successful in cardiac deformation modeling [34].

To the best of our knowledge, the only study that investigated the capability of FIS in finding a geometric transformation for image registration is [25]. Nevertheless, in [25], each pixel of the 2D image belonged to a fuzzy region and the affine transformation was considered for describing corresponding pixel transformation. The parameters of the affine transformation were computed through a fuzzy inference system. However, the problem definition for 3D TTE images is different from what presented in [25]. We use the positions of some relevant features for generating several fuzzy rules. Then, the obtained fuzzy rules are exploited for motion estimation. We believe that the proposed framework can improve the registration of the 3D TTE images through simplifying the system's complexity, reducing computation time and cost, and enabling interpretability of the registration results.

The remainder of the paper is organized as follows. In Section 2, the proposed algorithm for estimating cardiac motion is described in details. Section 3 presents the data description, evaluation metrics and implementation details. Experimental results are given in Section 4. In Section 5, some discussions on the proposed method and the obtained results are provided. Finally, Section 6 draws conclusions.

\section{Methodology}

\subsection{The fuzzy feature-based registration framework}

The proposed fuzzy feature-based registration framework was implemented in two main phases namely: 1) feature selection and, 2) fuzzy image registration. Figure 1 shows how an example fuzzy feature-based registration (FFR) task was performed during the first two phases. After having extracting image features using the 3D SIFT descriptor, fuzzy image registration computed a geometric transformation between the two corresponding feature values in two consecutive frames. At the end of the registration phase, a set of fuzzy rules was computed for every two consecutive frames.

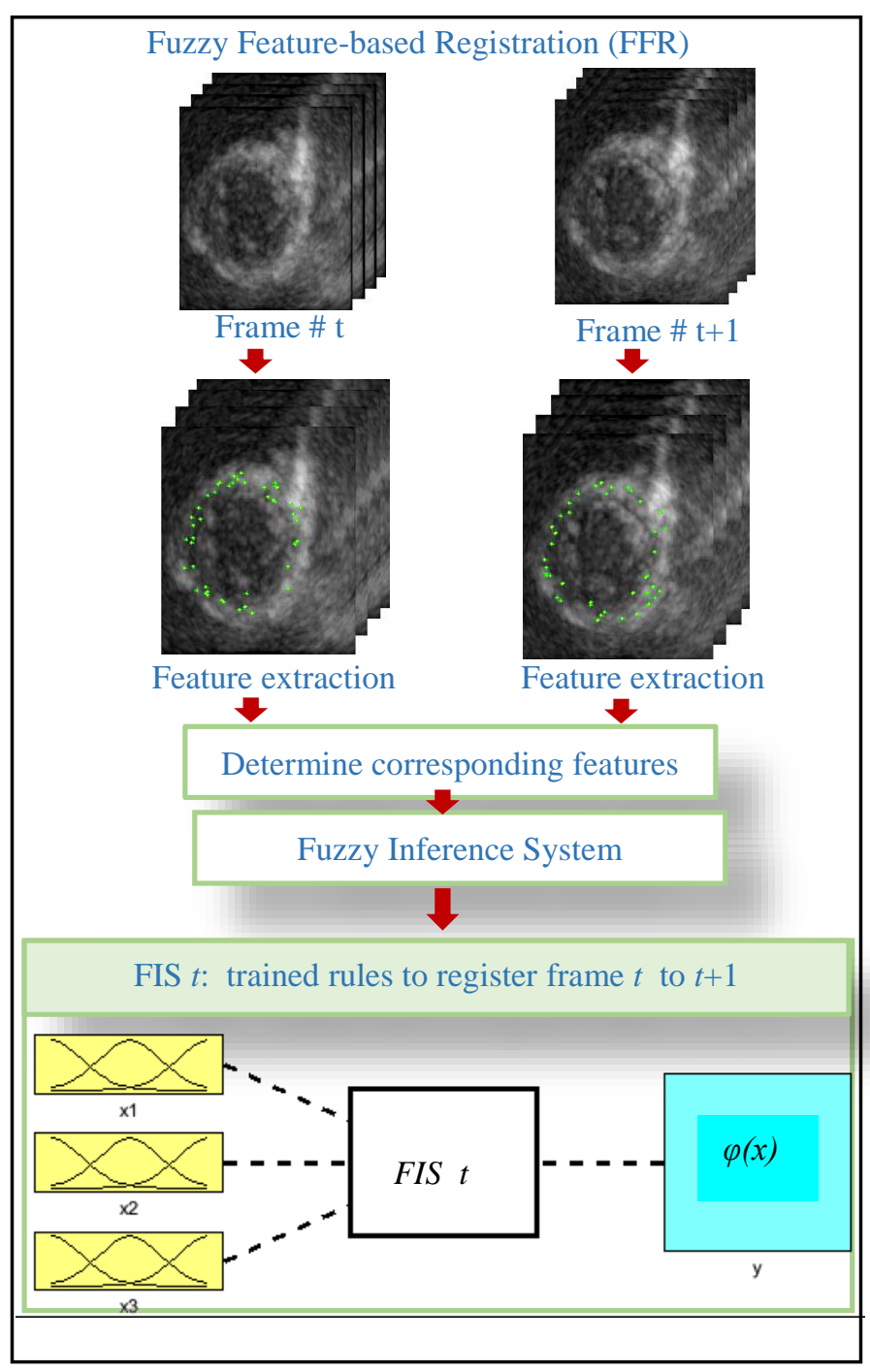

Figure 1. Block diagram of the proposed Fuzzy Feature-based Registration (FFR) algorithm. 


\subsubsection{Feature Extraction}

The first step of the feature-based registration method was to find features that are well-suited to represent the image properties. Since finding relevant key points is essential for the successful performance of the proposed registration algorithm, using an efficient feature extraction technique is of importance to ensure that the main image characteristics are captured.

There is a consensus among researchers that the SIFT descriptor performs best on key point extraction. Key points are the local maxima achieved by maximizing the Laplacian of the Gaussian function (LOG):

$$
l(\mathbf{x}, \sigma)=I(\mathbf{x}) * \Delta g_{\sigma}(\mathbf{x})
$$

where $\mathbf{x}=\left[x_{1} x_{2} x_{3}\right]$ contains the locations of the key points in 3D space and $\sigma$ represents the parameters of the Gaussian function. This convolution was approximated by the Difference of the Gaussian function (DoG):

$$
d(\mathbf{x}, \sigma)=I(\mathbf{x}) *\left(g_{\sigma+\delta}(\mathbf{x})-g(\mathbf{x})\right)
$$

where $\delta$ is a small value. Consequently, key points are the extrema of the DoG. In the next step, each key point should be described by a feature vector. A feature vector or key point descriptor was extracted through the following steps:

1. A spherical window with a radius of $2 \sigma$ was considered around each key point.

2. To have an orientation-invariant feature, the windowed image was rotated by the inverse of the key point's orientation.

3. The spherical window on the first step was divided into $4 \times 4 \times 4$ cubic sub-regions.

4. For each sub-region, a separate histogram was calculated.

5. The following formula computes the contribution of each voxel in the window:

$$
\lambda_{i} \exp \left(-\frac{\|\mathbf{x}-\mathbf{k}\|^{2}}{2 \sigma^{2}}\right) \prod_{i=1}^{n}\left(1-\frac{\sigma}{\sqrt{2}}\left|\mathbf{x}_{i}-\mathbf{z}_{i}\right|\right)
$$

where $\mathbf{K}$ is the key point's location, $\mathbf{z}$ is the subregion's center; and $\left(\lambda_{1}, \lambda_{2}, \lambda_{3}\right)$ are the barycentric coordinates of the key point. The exponential term is related to the spherical window, and the product term is the trilinear interpolation of weight for $\mathbf{z}$ [35].

\subsubsection{Fuzzy Image Registration}

Computing the transformation between the corresponding points is a major step in the registration process. As such, we first found the relevant key points in two consecutive frames. Then, the transformation between the corresponding key points was determined. The main novelty of this paper is to express each key point in the source image as a linear combination of the relevant key point in the reference image.
Consider $S_{1}$ and $S_{2}$ as the key point sets in the reference and source images, respectively. Let $\mathbf{y}_{1 S_{2}} \in S_{2}, \mathbf{y}_{2 S_{2}} \in S_{2}$ be the nearest and the second nearest key points to $\mathbf{x} \in S_{1}$ and $d(\mathbf{x}, \mathbf{y})$ be the Euclidean distance between $\mathbf{X}$ and $\mathbf{y}$. Lowes [31] defined a matching score which has become convention:

$g\left(\mathbf{x}, S_{2}\right)=\frac{d\left(\mathbf{x}, \mathbf{y}_{1 S_{2}}\right)}{d\left(\mathbf{x}, \mathbf{y}_{2 S_{2}}\right)}$

This matching score expresses that $\mathbf{x}$ and $\mathbf{y}_{1 S_{2}}$ can be corresponding key points, if $\mathbf{x}$ is much closer to $\mathbf{y}_{1 S_{2}}$ than to any other key points (i.e., when the matching score is below some threshold $\eta$ ). In order to have a bijective matching, i.e., the matches from $S_{1}$ to $S_{2}$ are the same as the matches found from $S_{2}$ to $S_{1}$; we performed the matching in both directions. Subsequently, there was a rejection in the uni-directional missing matches (i.e., $g>\eta$ ) or the ones that were not equal in both directions.

Computing a geometric transformation between the correspondence points can subsequently complete the registration task. We considered this step as a fuzzy modeling or a fuzzy identification problem.

Fuzzy modeling is composed of two main parts: 1) structure modeling and, 2) parameter identification [27]. The former part deals with rule numbers and input variables, while the latter part optimizes the location and shape of fuzzy sets in the premise and consequent parts of the fuzzy rules [27]. Due to simplicity and great potential of TSK in modeling, we used the TSK model for structure modeling [26]. A TSK fuzzy model with Multiple Inputs Single Output (MISO) is represented in the following form:

$$
\begin{aligned}
& \text { If } u_{1} \text { is } \mathscr{F}_{1} \text { and .... and } u_{n} \text { is } \mathscr{F}_{n} \\
& \text { then } v=\varphi\left(u_{1}, \ldots, u_{n}\right)
\end{aligned}
$$

where $n$ is the dimension of the input space, $u_{i}$ is the $i^{\text {th }}$ component of sample $\mathbf{u}$ and $\mathscr{F}_{i}, i=1, \ldots, n$ is a fuzzy set. In (5), $\varphi$ is any crisp function (i.e., a function with an exact output value) but is often chosen to be polynomial. If a firstorder polynomial is used, the resulting fuzzy inference is called the first-order TSK fuzzy model. A sample fuzzy rule of the first-order TSK system can thus be written as:

$$
\begin{array}{r}
\text { If } u_{1} \text { is } \mathscr{F}_{1} \text { and .... and } u_{n} \text { is } \mathscr{F}_{n} \\
\text { then } v=p_{0}+p_{1} u_{1}+\ldots+p_{n} u_{n}
\end{array}
$$

Each rule of the above fuzzy model has a crisp value in what follows and the overall output is the weighted average of the output of all rules.

Given these definitions, let $\left(\mathbf{x}_{1}, y_{1}\right), \ldots,\left(\mathbf{x}_{M}, y_{M}\right)$ $\mathbf{x}_{m} \in \square^{n}, y_{m} \in \square, m=1, \ldots, M$ ) be the $M$ input-output pairs of a first-order TSK model ( $M$ is the number of matched 
key points). For our problem, input samples $\left(\mathbf{x}_{m}\right)$ are the 3D positions of the key points in the reference image while output samples are one component of the corresponding key point in the source image. Since the MISO fuzzy system is considered to model a geometrical transformation in 3D, for each dimension in the source image a separate MISO system was defined.

Assume that the fuzzy system has R rules. The sample fuzzy rule of (6) is represented again for the $r^{\text {th }}$ rule [27] as:

$$
\begin{array}{r}
\text { If } x_{1} \text { is } F_{1}^{r} \text { and ... and } x_{n} \text { is } F_{n}^{r} \\
\text { then } \hat{y}^{r}=p_{0}^{r}+p_{1}^{r} x_{1}+\ldots+p_{n}^{r} x_{n}
\end{array}
$$

where $\hat{y}^{r}$ is a crisp value defined by $p_{i}^{r} \in \square$ parameters; and $F_{i}^{r}, i=1, \ldots, n$ is the membership function of the $r^{\text {th }}$ premise's fuzzy rules. Although the parameters of the membership functions can be optimized using an optimization algorithm, for the sake of simplicity, we set the premise's membership function to be a constant. The $r^{\text {th }}$ rule strength, $\omega^{r}$ , can be determined by each rule's weight through the product tnorm as:

$$
\omega^{r}\left(\mathbf{x}_{m}\right) \square \Lambda_{i \in\{1, . ., n\}} F_{i}^{r}\left(x_{i m}\right)=\prod_{i \in\{1, . ., n\}} F_{i}^{r}\left(x_{i m}\right)
$$

for $r=1, \ldots, R ; \quad \forall \mathbf{x}_{m} \in U_{1} \times \ldots \times U_{n}, U_{1} \in \square$.

The normalized strength, $v^{r}$, can then be defined as [27]:

$$
v^{r}\left(\mathbf{x}_{m}\right) \square \frac{\omega^{r}\left(\mathbf{x}_{m}\right)}{\sum_{i=1}^{R} \omega^{i}\left(\mathbf{x}_{m}\right)} \quad, \sum_{k=1}^{r} v^{k}\left(\mathbf{x}_{m}\right)=1
$$

The TSK fuzzy model performs a mapping $\hat{y}: U_{1} \times \ldots \times U_{n} \rightarrow \Psi$ with $\quad U_{i} \subset \square \quad$ and $\Psi \subset \square$. Considering the Larsen fuzzy inference system (product) and weighted output, the overall output can be calculated with the following formula:

$$
\hat{y}(\mathbf{x})=\frac{\sum_{r=1}^{R} \omega^{r}(\mathbf{x}) \cdot \hat{y}^{r}(\mathbf{x})}{\sum_{r=1}^{R} \omega^{r}(\mathbf{x})}
$$

The goal of the fuzzy model is to estimate $\hat{y}\left(\mathbf{x}_{m}\right)$ with (10) in order to be close to the related output in the measurement data $\left(y_{m}\right)$. In other words, consequent parameters should be optimized by minimizing the global modeling error:

$$
\|\vec{\varepsilon}\|^{2} \square\|\vec{y}-\overrightarrow{\hat{y}}\|^{2}
$$

where $\quad \vec{y} \square\left(y_{1}, \ldots, y_{m}\right)^{T}, \overrightarrow{\hat{y}} \square\left(\hat{y}_{1}, \ldots, \hat{y}_{m}\right)^{T}$ with

$\hat{y}_{m} \square \hat{y}\left(\mathbf{x}_{m}\right)$ for $m=1, \ldots, M$.
Let the matrix $A$ be of size $M \times(n+1) \cdot R$ is defined in order to solve the optimization problem (11). From (7)-(9), the matrix A can be represented as [27]:

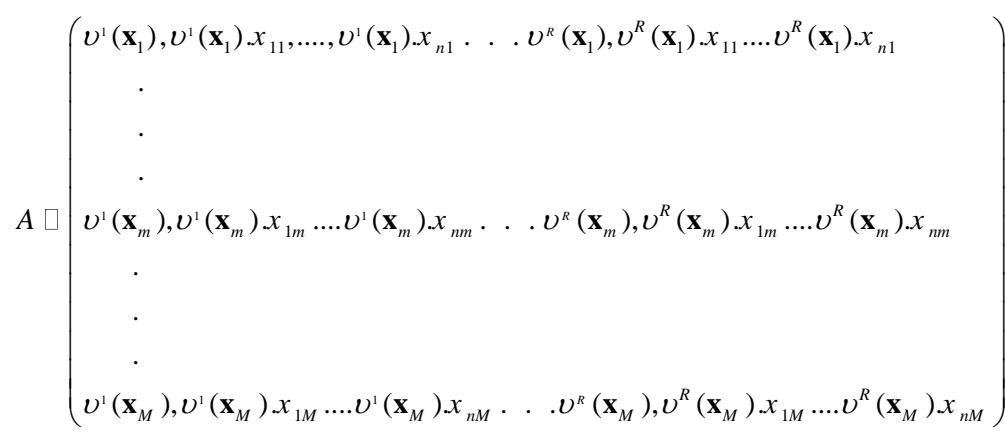

By considering the consequent parameter vector as $\vec{p} \square\left(p_{0}^{1} p_{1}^{1} \ldots p_{n}^{1} \ldots p_{0}^{R} p_{1}^{R} \ldots p_{n}^{R}\right)^{T}, \quad$ the model's output can be expressed with $\overrightarrow{\hat{y}}=A \cdot \vec{p}$. As the membership functions were assumed to be constants, the only variables of the optimization problem (11) are the parameter vectors, $\vec{p}$. Consequently, the least square solution of the optimization problem (11) gives the optimum response:

$$
\begin{aligned}
& \frac{\partial\|\vec{\varepsilon}\|^{2}}{\partial \vec{p}}=0 \Rightarrow \frac{\partial\left((\vec{y}-A \vec{p})^{T}(\vec{y}-A \vec{p})\right)}{\partial \vec{p}}=0 \Rightarrow \\
& -A^{T}(\vec{y}-A \vec{p})=0 \Rightarrow A^{T} \vec{y}=A^{T} A \vec{p} \Rightarrow \\
& \vec{p}=\left(\left(A^{T} A\right)^{-1} A^{T}\right) \vec{y}
\end{aligned}
$$

Using the explained fuzzy approach, the non-rigid optimization problem was thus solved by singular value decomposition (SVD)[27]. By computing the parameter vectors, $\vec{p}$, the complete set of fuzzy rules were determined and for every two consecutive frames, a set of fuzzy rules were created.

The premise of fuzzy rules includes $n$ clauses, which are input variables in each dimension. Given that the proposed registration method was designed for $3 \mathrm{D}$ echocardiographic images, each fuzzy rule was composed of three fuzzy clauses. Moreover, different strategies have been used for rule number computation. The conventional procedure is uniform gridding of the input space. However, it is possible to determine rule numbers through an optimization problem by incrementally increasing rule numbers to minimize the global modeling error.

\subsection{Fuzzy Motion Estimation}

The key point-based motion estimation can be finalized by computing the displacement field in all voxels based on the motion estimated in the key points. In other words, a dense displacement field should be generated from a sparse one. Interpolation could be the easiest way to obtain such a displacement field that is also smooth. However, using fuzzy rules empowers the motion estimation system to calculate the 
motion of each arbitrary point from the trained fuzzy rules. The fuzzy motion estimation is summarized in Figure 2.

\subsubsection{Primary Motion Estimation}

The temporal variable $t$ completes the proposed motion estimation framework. Hereto, $\hat{\mathbf{x}}(t)=\left[\hat{x}_{1}(t) \hat{x}_{2}(t) \hat{x}_{3}(t)\right]$ is a key point's position at time $t$. From the registration phase, $T-1$ sets of fuzzy rules were determined, where $T$ is the total number of frames in the cardiac cycle. The trained fuzzy rules at the initial frame and at time $t$ are represented as:

\section{FIS 1:}

$\left\{\begin{array}{l}\text { Rule 1:If } x_{1}(1) \text { is } F_{1}^{1} \& \ldots . \& x_{n}(1) \text { is } F_{n}^{1} \\ \text { then } \hat{x}_{1}(2)=p_{0}^{1}+p_{1}^{1} x_{1}(1)+\ldots+p_{n}^{1} x_{n}(1) \\ \text {. } \\ \text { Rule } R \text { :If } x_{1}(1) \text { is } F_{1}^{R} \& \ldots \text { \& } x_{n}(1) \text { is } F_{n}^{R} \\ \text { then } \hat{x}_{1}(2)=p_{0}^{R}+p_{1}^{R} x_{1}(1)+\ldots+p_{n}^{R} x_{n}(1)\end{array}\right.$

FIS $t$ :

$\left\{\begin{array}{l}\text { Rule 1:If } \hat{x}_{1}(t) \text { is } F_{1}^{1} \& \ldots \text { \& } \hat{x}_{n}(t) \text { is } F_{n}^{1} \\ \text { then } \hat{x}_{1}(t+1)=p_{0}^{1}+p_{1}^{1} \hat{x}_{1}(t)+\ldots+p_{n}^{1} \hat{x}_{n}(t) \\ \cdot \\ \text { Rule } R: \text { If } \hat{x}_{1}(t) \text { is } F_{1}^{R} \& \ldots \text { \& } \hat{x}_{n}(t) \text { is } F_{n}^{R} \\ \text { then } \hat{x}_{1}(t+1)=p_{0}^{R}+p_{1}^{R} \hat{x}_{1}(t)+\ldots+p_{n}^{R} \hat{x}_{n}(t)\end{array}\right.$

In the first frame, the motion tracking procedure started with a set of landmarks which were fed to FIS 1 to estimate the new positions in the next frame. The estimated positions were computed by trained $\vec{p},(13)$. Afterwards, the post-processing block (explained below) corrected inaccurate estimations which continued till the end of the cardiac cycle.

\subsubsection{Post-processing}

The post-processing block was designed to modify inaccurate estimations to avoid error propagation and accumulation through the cardiac cycle. Such estimates were discovered based on the fact that the myocardium does not deform significantly between two consecutive frames.

Let $\mathbf{d}_{1} \square \hat{\mathbf{x}}(t+1)-\hat{\mathbf{x}}(t)$ be the displacement between the estimated 3D voxel positions by the FIS rules at time $t$, i.e., the estimated 3D voxel position by the FIS rules at time $t-1$. $\hat{\mathbf{x}}(t+1)$ was modified through the first modification rule (14).

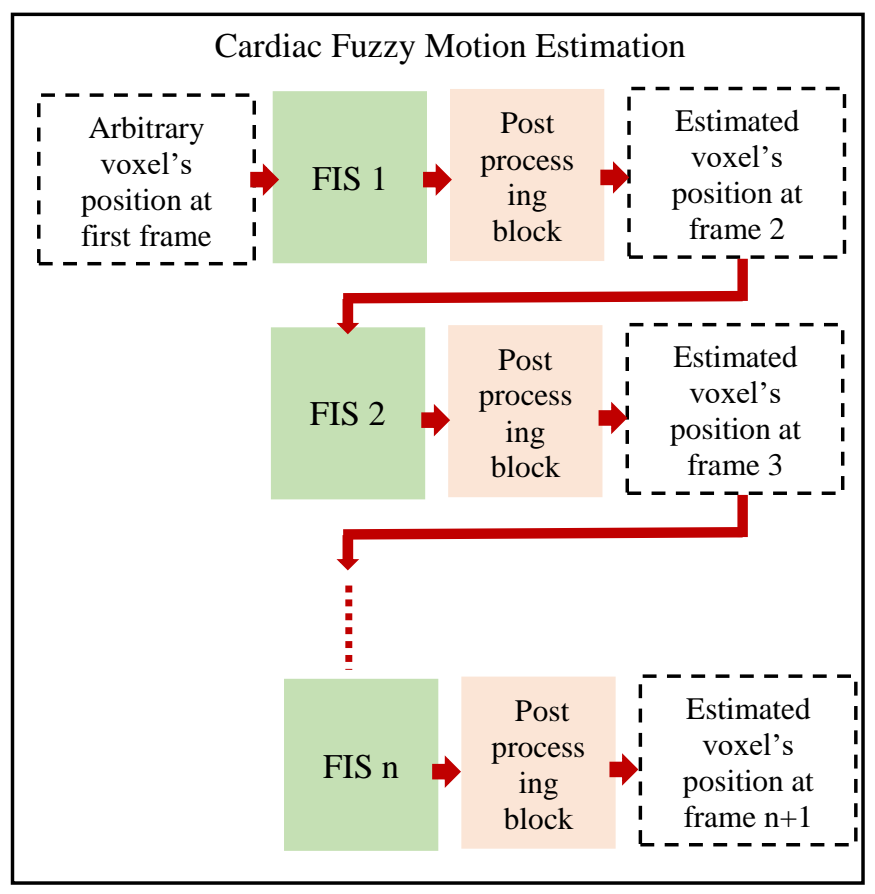

Figure 2. Block diagram of the proposed cardiac fuzzy motion estimation approach

if $\left\|\mathbf{d}_{1}\right\|>\zeta$ then $\widehat{\mathbf{x}}(t+1)=\bar{m} \hat{\mathbf{x}}(t)+\bar{b}$

where $\bar{m}$ and $\bar{b}$ were determined by solving the following optimization problem:

$$
\begin{array}{ccc}
\min & \|\hat{\mathbf{x}}(t+1)-\hat{\mathbf{x}}(t)\| & \\
\text { s.t. } & b_{i} \neq 0 & \forall i=1,2,3 \\
-2 \leq b_{i} \leq 2 & \forall i=1,2,3 \\
0.9 \leq m_{i} \leq 1 & \forall i=1,2,3
\end{array}
$$

Let $\mathbf{d}_{2} \square \hat{\mathbf{x}}(t+1)-\hat{\mathbf{x}}(t)$; the modified position could be accepted through the second modification rule (16).

$$
\begin{cases}\text { if } \quad\left\|d_{2}\right\|<\zeta \text { then } \quad \hat{\mathbf{x}}(t+1)=\hat{\mathbf{x}}(t+1) \\ \text { if } \quad\left\|d_{2}\right\| \geq \zeta \text { then } \quad \hat{\mathbf{x}}(t+1)=\hat{\mathbf{x}}(t)\end{cases}
$$

Like the FIS rules, at the modification phase, the new voxel position was established as a linear combination of the previous voxel's position. Contrary to the FIS rules, the modified position has an independent linear relation with the initial position in each dimension.

\section{EXPERIMENTS}

\subsection{Data Description}

\subsubsection{Synthetic Database}


We used the STRAUS database in order to evaluate the performance of the proposed registration algorithm. STRAUS is an in-silico 3D ultrasound database, which was introduced in [36]. It includes eight synthetic volumetric ultrasound sequences of a cardiac model mimicking one healthy subject and seven pathological cases. The pathological cases were divided into two categories: ischemic cases, which had an occlusion in the coronary arteries, and dilated cardiomyopathy cases.

In the ischemic sequences, the occlusion occurred in the proximal or distal parts of the left anterior descending coronary artery (LADprox, LADdist), the left circumflex coronary artery (LCX), and the right coronary artery (RCA). The three dilated cardiomyopathy cases contain a synchronous activation pattern (sync) and two dyssynchronous patterns due to left branch bundle block (LBBBsmall and LBBBlarge) [37].

The ground truth mesh points in anatomical directions were provided for all sequences: 30 points in the longitudinal (L), 25 points in the circumferential (C), and 3 points in the radial (R) directions. The time resolution for all sequences was 34 frames/s [37]. The volume data of each frame was $224 \times 176 \times 208$ voxels with a voxel size of $0.7 \times 0.9 \times 0.6 \mathrm{~mm}^{3}$. The full database is publicly available at ${ }^{l}$.

\subsubsection{In-vivo Data}

In order to evaluate the capability of the proposed registration method in-vivo, we used some sequences provided in the CETUS project [38]. CETUS was a segmentation TTE challenge including echocardiographic images of three groups: healthy, ischemic and dilated cardiomyopathy patients. The entire database contains 45 sequences with 15 subjects that were considered as training set. For training images, the endocardium border on End-Diastolic (ED) and End-Systolic (ES) frames are available as reference [39]. In this study, we used these training images as they had reference points for evaluation available.

\subsection{Performance Assessment}

\subsubsection{Tracking Accuracy}

Let $\mathbf{x}^{i}(t)$ be the 3D position of $i^{\text {th }}$ ground-truth mesh point and $\hat{\mathbf{x}}^{i}(t)$ represents the $3 \mathrm{D}$ position of the estimated point at frame $t$. The tracking error was defined as: $e^{i}(t) \square \mathbf{x}^{i}(t)-\hat{\mathbf{x}}^{i}(t)$.

The tracking algorithm was initiated by the ground-truth point cloud of the first frame and tracked incrementally throughout the cardiac sequence. Therefore, the error which may occur between two consecutive frames can be accumulated and affect the next estimation. Besides the global tracking error (17), we also evaluated the global error in the physiologic L, R, and C directions separately. Moreover, the local error was computed based on the standard AHA left ventricular segmentation model. In this model, the LV is split into 17 segments, which are illustrated in Figure 3.

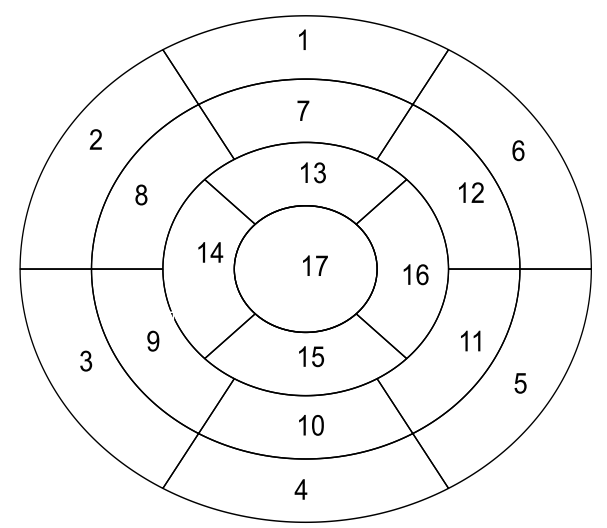

Figure 3. Standard AHA segmentation

\subsubsection{Strain Accuracy}

Strain analysis is a well-established technique to quantify regional myocardial motion and deformation in clinical practice. It was, therefore, logic to evaluate the efficacy of the proposed registration method in terms of the strain estimation accuracy. Strain was measured as the relative change in distance between two neighboring mesh nodes in the three physiologic directions. Let $\ell_{n}(t)$ be the distance between two consecutive nodes at time $t$ with $n \in\{L, C, R\}$. Strain was then defined as:

$\varepsilon_{n}(t)=\ell_{n}(t) / \ell_{n}(0)-1$

The full strain profile was computed for all sequences in comparison with the reference one, and the relative error (19) was measured.

$\sum_{t} \frac{\mid \text { estimated_strain }(t)-\text { reference_strain }(t) \mid}{\sum_{t} \mid \text { reference_strain }(t) \mid}$

\subsubsection{Contour metrics}

Given that ground truth mesh points are not available in the CETUS database, it was not possible to measure the tracking performance using the above-mentioned tracking or strain accuracy criteria. As such, contour metrics were defined to show the capacity of the proposed method. Hereto, the ground truth ED endocardial contour was tracked to ES with the proposed algorithm. This tracked ES contour was subsequently contrasted with the ground truth ES contour using the following indices:

- Dice Similarity Index: this metric is used to compute the overlapping region between the two volumes: 


$$
\text { Dice }=\frac{2\left(V_{e s t} \cap V_{r e f}\right)}{V_{e s t}+V_{r e f}}
$$

where $V_{\text {est }}$ refers to the volume obtained by tracking the ED contour and $V_{r e f}$ is the ground truth ES volume.

- Mean Surface Distance: the mean Euclidean distance is distance between every point of the tracked ES surface ( $S_{e s t}$ ) to the closest point on the ground truth surface [40].

\subsection{Implementation Details}

Extracted key points were limited to the myocardium based on the ground truth meshes. The relevant key points were determined by a matching threshold $\eta=0.8$. We used a Gaussian membership function in the FIS rules with three uniform divisions in each dimension. Finally, inaccurate estimations were adjusted by the modification rules with a deformation threshold $\zeta=2$.

\section{Results}

\subsection{Tracking Accuracy}

The computed tracking errors for the synthetic database were compared with two categories of alternative registration methods and the results are summarized in Table 1 and Table 2. Table 1 relates the proposed method (FFR) to well-known and successful non-rigid registration methods including Radial Basis Function (RBF) transformation, thin-plate spline (TPS) transformation, and Coherent Point Drift (CPD) [41] while Table 2 compares it with state-of-the-art methods in 3D speckle tracking.

Table 1 shows that the proposed fuzzy algorithm (FFR) outperforms other conventional non-rigid registration methods in cardiac motion estimation. In addition to RBF, CPD and TPS, the RBF network presented in [42] was also tested. Given that the RBF network is equivalent to the zero-order TSK model [43], [44], two conclusions can be drawn. First, the zero-order TSK model was comparable with conventional non-rigid point registration algorithms. Second, the first-order TSK outperformed zero-order TSK for 3D speckle tracking.

Table 1. Global Tracking Error in $\mathrm{mm}$ for the STRAUS database

\begin{tabular}{lccc} 
Algorithm & 5-th percentile & median & 95-th percentile \\
\hline CPD & 0 & 2.98 & 7.2 \\
TPS & 0 & 1.4 & 4.9 \\
RBF & 0 & 1.39 & 4.89 \\
RBF Network & 0 & 1.34 & 4.75 \\
FFR & $\mathbf{0 . 2 5}$ & $\mathbf{1}$ & $\mathbf{2 . 5 2}$ \\
\hline
\end{tabular}

In addition to perform 3D tracking with high accuracy, the proposed FFR algorithm has two important features namely interpretability and simplicity. As explained in Section 2, there is no need to create and calculate a dense displacement field as a consequence of using a rule-based system. In such a system, tracking each voxel is obtained by feeding the voxel spatial information in the series of rules.

Table 2 presents the tracking errors for different cardiac morphologies. In the STRAUS database, healthy and ischemic subjects have normal LV morphology while the other subjects have a dilated ventricle [37]. The results show that our fuzzy system was comparable with Block Matching (BM), especially for the subjects with dilated geometry. Besides, in terms of the 95-th percentile of the error, FFR outperformed the RF-based BM (RFBM) [45]. Note that the BM matching method has been widely used for cardiac motion estimation both for academic and commercial purposes.

Table 2. Global Tracking Errors for the STRAUS database in $\mathrm{mm}$ [5th percentile, Median, 95-th percentile] based on geometry

\begin{tabular}{lcccc} 
Algorithm & Full database & Normal Geometry & Dilated Geometry \\
\hline FFR & {$[0.25 ; 1.00 ; 2.52]$} & {$[0.26 ; 1.00 ; 2.56]$} & {$[0.21 ; 0.99 ; 2.38]$} \\
BM & {$[0.26 ; 0.90 ; 2.46]$} & {$[0.26 ; 0.86 ; 2.11]$} & {$[0.25 ; 0.99 ; 3.19]$} \\
RFBM & {$[0.09 ; 0.72 ; 4.33]$} & {$[0.10 ; 0.72 ; 4.40]$} & {$[0.07 ; 0.73 ; 4.23]$} \\
\hline
\end{tabular}

The tracking error was also calculated in the anatomical directions. The box plots of the tracking errors in the L, C, and $\mathrm{R}$ directions are illustrated in Figure 4. By evaluating these anatomical errors, the results from Table 2 are confirmed. Taking the median of the errors into account, the proposed fuzzy system performed as well as the state-of-the-art methods. Another observation that can be made from the directional error analysis is that the proposed method outperformed RFBM in the longitudinal direction.

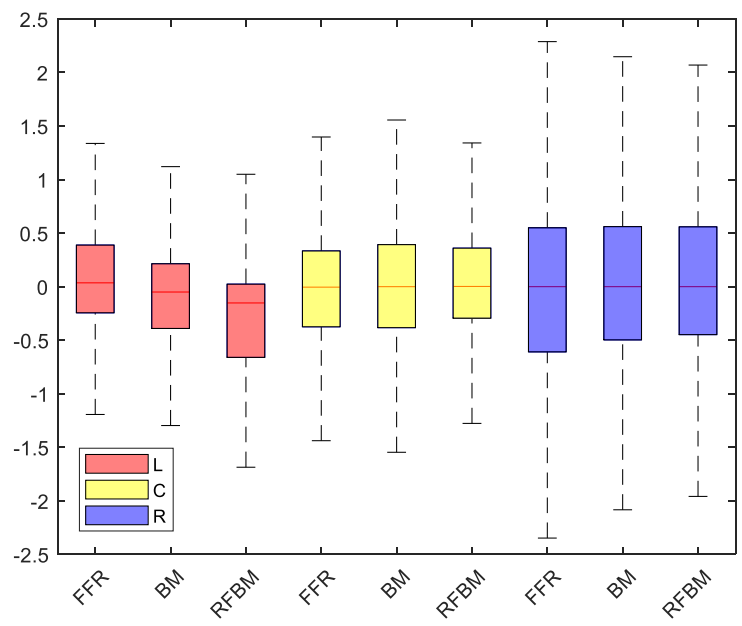

Figure 4. Boxplot tracking errors in the L, C and $\mathrm{R}$ directions for the examined registration methods.

In another experiment, the tracking errors were analyzed regionally (i.e., per ventricular segment). Figure 5 shows the bulls-eye plots of the regional tracking errors by applying the fuzzy system algorithm for the different cardiac geometrics. It can be seen that the registration algorithm led to large tracking errors for some of the basal segments. This can be explained by low image quality of the segments that are far from the ultrasound probe. Given that the performance of the proposed 
fuzzy registration algorithm is highly dependent on the features that are extracted from the myocardial images, it is expected that the quality of the features extracted from the low-resolution regions is worse and as a consequence, the tracking error for these regions is larger.
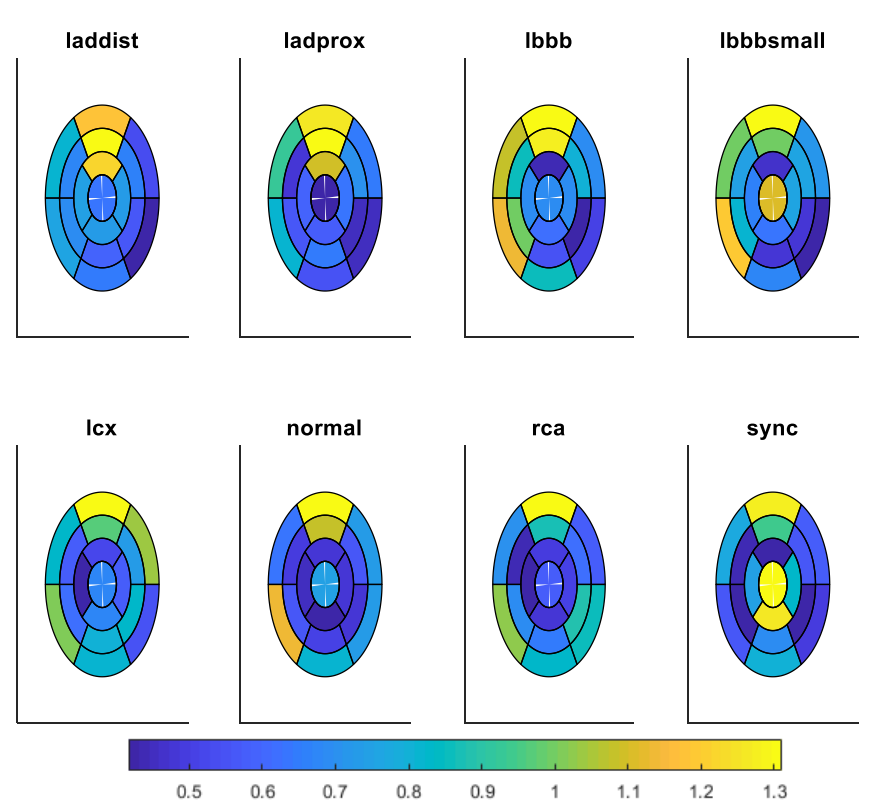

Figure 5. Bulls-eye plots of the regional tracking errors of the proposed registration method

\subsection{Strain Estimation Accuracy}

The average values of the relative strain estimation errors for the synthetic database in the three anatomical directions are listed in Table 3 . The best performance is shown in bold in each column. The proposed fuzzy system outperformed the state-ofthe-art in the C-direction. Although FFR was not the best for L and $\mathrm{R}$ strain estimation, it showed to be competitive with the RFBM algorithm.

\subsection{Contour Metrics}

Applying the FFR algorithm on the training images of the CETUS database resulted in an average Dice metric of $0.77 \pm$ 0.19 and a mean distance equal to $3.03 \pm 1.6 \mathrm{~mm}$. This indicates an acceptable similarity between the volume obtained with our method and the reference volume on the ES frame. Figure 6 illustrates an example comparing the volume obtained by tracking the ED contour with the ground truth ES volume. Furthermore, mesh points obtained by tracking the ED contour from different 2D views are provided in Figure 7.

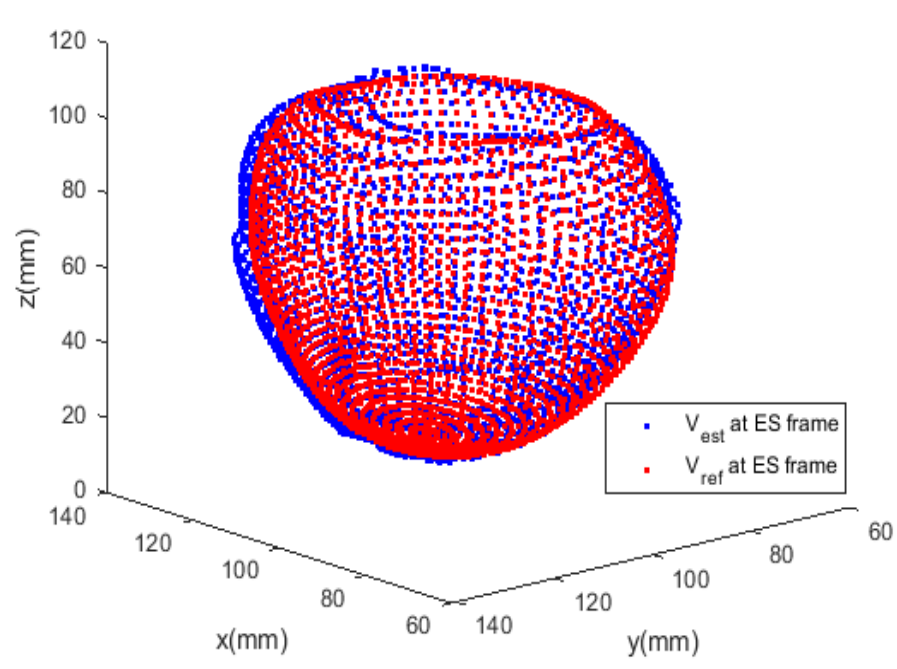

Figure 6. An example comparing the volume obtained by tracking the ED contour with the ground truth ES volume. $V_{e s t}$ refers to the volume obtained by tracking the ED contour and $V_{r e f}$ is the ground truth ES volume.

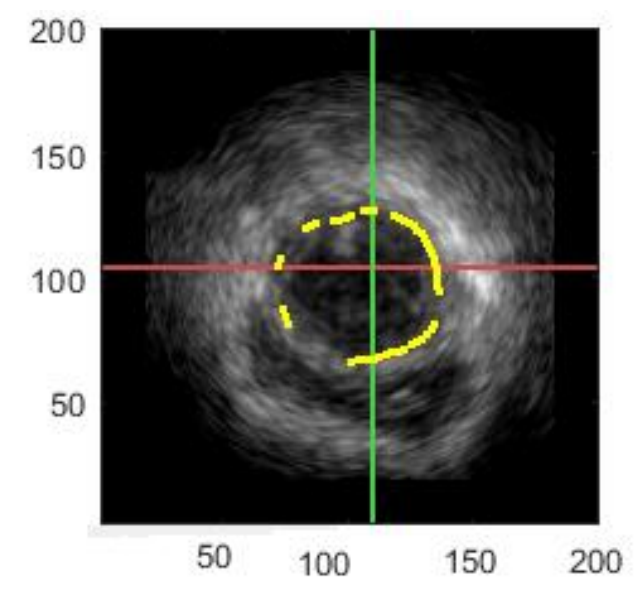

(a)

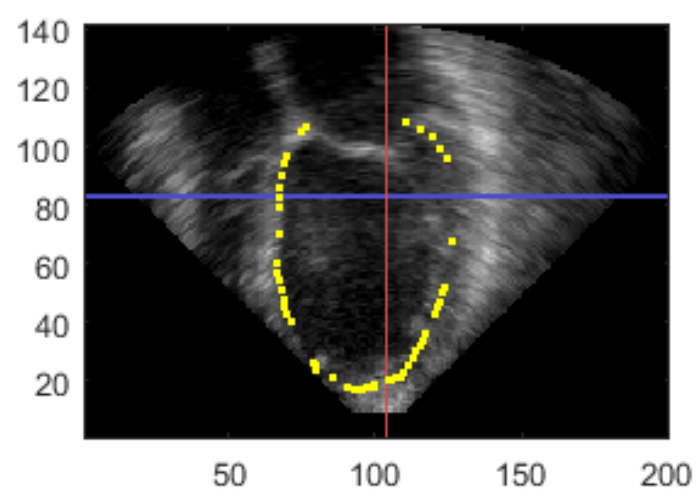

(b)

Figure 7. Mesh points obtained by tracking the ED contour to ES frame from different 2D views. 


\subsection{Computational Time}

For implementation of the proposed registration algorithm, most of the computational time was spent on the descriptor representation step. The whole algorithm was implemented in a MATLAB environment but for computational efficiency, it linked to $\mathrm{C}$ for the feature extraction part. The FFR algorithm required around $13 \mathrm{~min} /$ frame when implemented using a system with Core ${ }^{\mathrm{TM}} \mathrm{i}$, $3.3 \mathrm{GHz}$ CPU and $32 \mathrm{~GB}$ of RAM. As reported in [37], the BM algorithm required 129 s/frame when it was coded in MATLAB in an Intel i7, 6GB RAM notebook. In addition, the RFBM method needed $50 \mathrm{~min} /$ frame on a standalone Intel(R) Core i7 with 3.6GHz CPU and 32GB RAM [37].

Table 3. The relative strain error values for the STRAUS database (in $\%)$ between the estimated and references curve in three anatomical directions (mean \pm standard deviation). In each column, the best performance is shown in bold black font).

\begin{tabular}{cccc} 
Algorithm & L-Strain & C-Strain & R-Strain \\
\hline FFR & $11.09 \pm 387.46$ & $\mathbf{0 . 8 2} \pm \mathbf{4 . 6 9}$ & $4.12 \pm 13.13$ \\
\hline BM & $\mathbf{8 . 6 1} \pm \mathbf{3 0 2 . 6 8}$ & $1.11 \pm 7.88$ & $\mathbf{1 . 9 0} \pm \mathbf{9 . 6 3}$ \\
\hline RFBM & $32.52 \pm 670.01$ & $2.29 \pm 24.71$ & $4.00 \pm 40.07$ \\
\hline
\end{tabular}

\section{DISCUSSION}

This manuscript presents a conceptually new approach towards 3D cardiac motion estimation in echocardiography based on fuzzy logic. The proposed estimator showed to be competitive with the state-of-the-art on synthetic data in terms of accuracy. Although initial testing in-vivo showed the potential of this new approach, further optimization and refinement are required to make it a useful approach to be applied in the clinic. However, such further optimization fell outside the scope of the present paper that only wanted to test the feasibility of applying fuzzy logic for motion estimation in 3D echocardiography.

As mentioned in Section 2.1.2, we used uniform partitions in the premise of fuzzy rules where each rule's output needed four parameters to be determined. Therefore, with $n$ uniform partitions in the premise part, $4 n^{3}$ parameters had to be optimized using the least-squares optimization algorithm. For the learning method, a good ratio for setting the number of parameters with respect to input-output pairs is about $1 / 10$. On the other hand, the number of corresponding features between two frames was in the order of 1000. Consequently, three segmentations per dimension could be a reasonable assumption. Nevertheless, because the number of corresponding features for extracting different FIS varied from 619 to 3318, other rule numbers were also examined. Figure 8 demonstrates the tracking error versus an equal partition number and confirms the suitability of three partitions.

The first-order TSK is the first step of embedding image registration and motion estimation in a fuzzy system. In the future, we are going to express the consequent parts of rules with fuzzy functions instead of crisp ones. In addition, a more precise function which is matched with real cardiac motion based on physiology may lead to better results.
In addition to the ability of fuzzy systems for estimating the geometrical transformation, this theory has an excellent capability for finding similarities. This suggests that the corresponding feature points can be defined with fuzzy similarity criteria. However, we believe that the mentioned extension may lead to significant improvement in cardiac motion estimation even in real echocardiography.

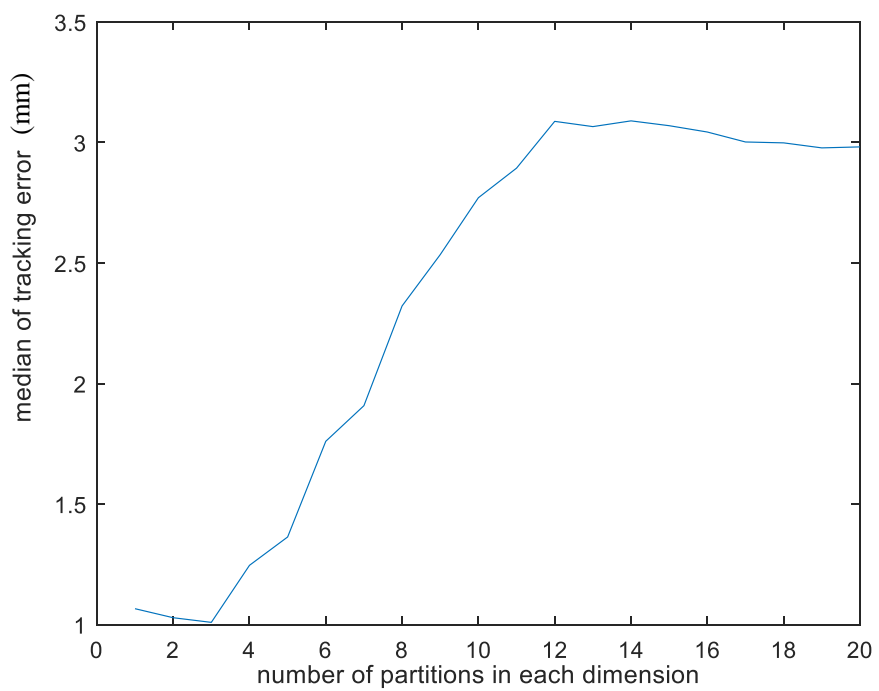

Figure 8. Median of the tracking error versus the number of partitions.

It should be mentioned that we compared our method on the available computers with those reported in literature run on the same STRAUS database. Re-implementing the alternative methods would not only be time consuming but would also remain biased as our implementation may differ from those reported in literature.

\section{CONCLUSION}

In this paper, an algorithm was proposed for cardiac motion estimation using fuzzy feature-based rules in 3D TTE images. The fuzzy rules were generated for the extracted spatial features between two consecutive frames. The generated rules were exploited to estimate the motion of any arbitrary voxel throughout the cardiac cycle. The experiments on a synthetic database showed that the proposed algorithm outperformed some of the conventional non-rigid image registration methods. Moreover, its performance was comparable with BM-based 3D cardiac motion estimation algorithms both in terms of tracking error and strain accuracy. Furthermore, the proposed algorithm yielded the results on the CETUS database indicating its potential for being used in the clinical application. Two main conclusions can be drawn from the results obtained with the proposed fuzzy registration algorithms: 1) the SIFT descriptor encodes essential spatial features for 3D echocardiographic motion estimation and, 2) the modified first-order TSK fuzzy rule system is successful in aligning accurate information to estimate cardiac motion. 


\section{Acknowledgment}

The authors wish to express their appreciations to Aboozar Ghaffari for his kind advices.

\section{REFERENCES}

[1] D. K. Iakovidis, E. G. Keramidas, and D. Maroulis, "Fuzzy Local Binary Patterns for Ultrasound Texture Characterization, " in Image Analysis and Recognition, Berlin, Heidelberg: Springer Berlin Heidelberg, 2008, pp. 750-759.

[2] A. Fatemi, E. A. R. Berg, and A. Rodriguez-Molares, "Studying the Origin of Reverberation Clutter in Echocardiography: In Vitro Experiments and In Vivo Demonstrations," Ultrasound Med. Biol., vol. 45, no. 7, pp. 1799-1813, 2019, doi: 10.1016/j.ultrasmedbio.2019.01.010.

[3] M. Kumar, N. Stoll, K. Thurow, and R. Stoll, "Fuzzy Membership Descriptors for Images," IEEE Trans. Fuzzy Syst., vol. 24, no. 1, pp. 195-207, 2016, doi: 10.1109/TFUZZ.2015.2451706.

[4] P. Hurtik, I. Perfilieva, and P. Hodáková, “Fuzzy Transform Theory in the View of Image Registration Application," Commun. Comput. Inf. Sci., vol. 443 CCIS, no. PART 2, pp. 143-152, 2014, doi: 10.1007/978-3-319-08855-6_15.

[5] M. Wan, G. Yang, Z. Lai, and Z. Jin, "Feature extraction based on fuzzy local discriminant embedding with applications to face recognition," Comput. Vision, IET, vol. 5, no. 5, pp. 301-308, 2011, doi: 10.1049/iet-cvi.2011.0028.

[6] H. R. Tizhoosh, "Image thresholding using type II fuzzy sets, ” Pattern Recognit., vol. 38, no. 12, pp. 2363-2372, Dec. 2005, doi: 10.1016/j.patcog.2005.02.014.

[7] H. Mahmoud, F. Masulli, and S. Rovetta, "Featurebased medical image registration using a fuzzy clustering segmentation approach," Lect. Notes Comput. Sci. (including Subser. Lect. Notes Artif. Intell. Lect. Notes Bioinformatics), vol. 7845 LNBI, pp. 37-47, 2013, doi: 10.1007/978-3-642-38342-7_4.

[8] F. Li, Q. Shen, Y. Li, and N. Mac Parthaláin, "Handwritten Chinese character recognition using fuzzy image alignment," Soft Comput., vol. 20, no. 8, pp. 2939-2949, 2015, doi: 10.1007/s00500-015-1923$y$.

[9] J. S. Marques, "A fuzzy algorithm for curve and surface alignment,” Pattern Recognit. Lett., vol. 19, no. 9, pp. 797-803, 1998, doi: 10.1016/S0167-8655(98)00063-4.

[10] J. Tarel, N. Boujemaa, L. C. Cedex, and L. I. Ecole, A Fuzzy 3D Registration Method, no. August. 1995.

[11] Z. Xue, D. Shen, and K. E. Teoh, "An efficient fuzzy algorithm for aligning shapes under affine transformations, " Pattern Recognit., vol. 34, no. 6, pp. 1171-1180, 2001, doi: 10.1016/S00313203(00)00067-4.

[12] G. Wang, Z. Wang, Y. Chen, W. Zhao, and X. Liu, "Fuzzy Correspondences and Kernel Density Estimation for Contaminated Point Set Registration," in 2015 IEEE International Conference on Systems, Man, and Cybernetics, 2015, pp. 1936-1941, doi: 10.1109/SMC.2015.338.

[13] Yuefeng Zhang, "A fuzzy approach to digital image warping,” IEEE Comput. Graph. Appl., vol. 16, no. 4, pp. 34-41, Jul. 1996, doi: 10.1109/38.511850.

[14] A. Kolagunda, S. Sorensen, P. Saponaro, W. Treible, and $C$. Kambhamettu, "Robust Shape Registration using Fuzzy Correspondences, ” Feb. 2017.

[15] R. Gallea, E. Ardizzone, R. Pirrone, and O. Gambino, "Three-dimensional Fuzzy Kernel Regression framework for registration of medical volume data," Pattern Recognit., vol. 46, no. 11, pp. 3000-3016, 2013, doi: 10.1016/j.patcog.2013.03.025.

[16] A. Trist, "Fuzzy regularisation of deformation fields in image registration," Image (Rochester, N.Y.), vol. $d$, no. 08, pp. 1223-1230, 2008.

[17] A. Reh, A. Amirkhanov, J. Kastner, E. Gröller, and C. Heinzl, "Fuzzy feature tracking: Visual analysis of industrial 4D-XCT data, ” Comput. Graph., vol. 53, pp. 177-184, 2015, doi: 10.1016/j.cag.2015.04.001.

[18] S. CC and P. Kulkarni, "Region based Image Similarity using Fuzzy based SIFT Matching," Int. J. Comput. Appl., vol. 67, no. 3, pp. 47-50, 2013, doi: 10.5120/11379-6655.

[19] S. Susan, A. Jain, A. Sharma, S. Verma, and S. Jain, "Fuzzy match index for scale-invariant feature transform (SIFT) features with application to face recognition with weak supervision," IET Image Process., vol. 9, no. 11, pp. 951-958, 2015, doi: 10.1049/iet-ipr.2014.0670.

[20] C. Guo-rong, L. Shao-zi, W. Yun-dong, C. Shui-li, and S. Song-zhi, "Automatic registration of remote sensing images based on SIFT and fuzzy block matching for change detection," Comput. Intell., vol. 4, no. 5, pp. 874-885, 2011.

[21] T. Dong, T. Deng, J. Dai, W. Xie, J. Yang, and Q. Fu, "A fuzzy reasoning method for multi-views image registration,” J. Comput., vol. 8, no. 10, pp. 26892694, 2013, doi: 10.4304/jcp.8.10.2689-2694.

[22] F. Di Martino and S. Sessa, "Image matching by using fuzzy transforms, ” Adv. Fuzzy Syst., vol. 2013, 2013, doi: 10.1155/2013/760704.

[23] P. Zarychta, "Application of fuzzy image concept to medical images matching," Adv. Intell. Syst. Comput., vol. 762, pp. 27-38, 2019, doi: 10.1007/978-3-31991211-0_3.

[24] X. Lu, S. Zhang, W. Yang, and Y. Chen, "SIFT and shape information incorporated into fluid model for non-rigid registration of ultrasound images, " Comput. Methods Programs Biomed., vol. 100, no. 2, pp. 123131, 2010, doi: 10.1016/j.cmpb.2010.03.005.

[25] F. L. Chung, Z. Deng, and S. Wang, "An adaptive fuzzyinference-rule-based flexible model for automatic elastic image registration," IEEE Trans. Fuzzy Syst., vol. 17, no. 5, pp. 995-1010, 2009, doi: 10.1109/TFUZZ.2009.2020154.

[26] T. Tagaki and M. Sugeno, "Takagi Sugeno Modelling(1).pdf." pp. 116-132, 1985.

[27] M. Männle, "Identifying rule-based TSK fuzzy 
models," Proc. Eur. Congr. Intell. Tech. Soft Comput. (EUFIT 1999), Aachen, Ger. Elit. Found., pp. 286-299, 1999.

[28] S. J. Wu, "Affine TS-model-based fuzzy regulating/servo control design, ” Fuzzy Sets Syst., vol. 158, no. 20, pp. 2288-2305, 2007, doi: 10.1016/j.fss.2007.05.012.

[29] A. Kalhor, B. N. Araabi, and C. LUCAS, “A New Highorder Takagi-Sugeno Fuzzy Model Based on Deformed Linear Models," Amirkabir Int. J. Model. Identification, Simul. Control, vol. 42, no. 2, pp. 43-52, 2010, doi: 10.22060/MISCJ.2010.210.

[30] M. Herceg, M. Kvasnica, and M. Fikar, "Transformation of Fuzzy Takagi-Sugeno Models into Piecewise Affine Models," Rough Sets Intell. Syst. Paradig. Proc., vol. 4585, pp. 211-220, 2007.

[31] D. G. Low, "Distinctive image features from scaleinvariant keypoints, ” Int. J. Comput. Vis., pp. 91-110, 2004.

[32] Y. Ke and R. Sukthankar, "PCA-SIFT: A More Distinctive Representation for Local Image Descriptors, " pp. 2-9, 2004.

[33] Mahesh and M. V. Subramanyam, "Automatic feature based image registration using SIFT algorithm, ” 2012 3rd Int. Conf. Comput. Commun. Netw. Technol. ICCCNT 2012, no. July, 2012, doi: 10.1109/ICCCNT.2012.6396024.

[34] K. McLeod, C. Seiler, N. Toussaint, M. Sermesant, and $X$. Pennec, "Regional analysis of left ventricle function using a cardiac-specific polyaffine motion model," Lect. Notes Comput. Sci. (including Subser. Lect. Notes Artif. Intell. Lect. Notes Bioinformatics), vol. 7945 LNCS, pp. 483-490, 2013, doi: 10.1007/978-3642-38899-6_57.

[35] B. Rister, M. A. Horowitz, and D. L. Rubin, "Volumetric Image Registration From Invariant Keypoints,” IEEE Trans. Image Process., vol. 26, no. 10, pp. 4900-4910, Oct. 2017, doi: 10.1109/TIP.2017.2722689.

[36] M. Alessandrini et al., "A Pipeline for the Generation of Realistic 3D Synthetic Echocardiographic Sequences: Methodology and Open-Access Database, " IEEE Trans. Med. Imaging, vol. 34, no. 7, pp. 14361451, 2015, doi: 10.1109/TMI.2015.2396632.

[37] M. Alessandrini et al., "Detailed Evaluation of Five 3D Speckle Tracking Algorithms Using Synthetic Echocardiographic Recordings," IEEE Trans. Med. Imaging, vol. 35, no. 8, pp. 1915-1926, Aug. 2016, doi: 10.1109/TMI.2016.2537848.

[38] O. Bernard et al., "Challenge on Endocardial Threedimensional Ultrasound Segmentation ( CETUS )," Proc. MICCAI Chall. Echocardiogr. ThreeDimensional Ultrasound Segmentation, pp. 1-8, 2014.

[39] O. Bernard et al., "Standardized evaluation system for left ventricular segmentation algorithms in $3 D$ echocardiography," IEEE Trans. Med. Imaging, vol. 0062, no. c, pp. 1-1, 2015, doi: 10.1109/TMI.2015.2503890.

[40] D. Frisch, "point2trimesh - Distance between a point and a triangulated surface in 3D," 2021. [Online].
Available:

https://www.mathworks.com/matlabcentral/fileexchan ge/52882-point2trimesh-distance-between-point-andtriangulated-surface.

[41] A. Myronenko and Xubo Song, "Point Set Registration: Coherent Point Drift," IEEE Trans. Pattern Anal. Mach. Intell., vol. 32, no. 12, pp. 2262-2275, Dec. 2010, doi: 10.1109/TPAMI.2010.46.

[42] D. S. Broomhead and D. Lowe, "Radial basis functions, multi-variable functional interpolation and adaptive networks," no. 4148, 1988.

[43] H. C. Anderson, A. Lotfi, L. C. Westphal, and J. R. Jang, "Comments on 'Functional equivalence between radial basis function networks and fuzzy inference systems' [with reply], " IEEE Trans. Neural Networks, vol. 9, no. 6, pp. 1529-1532, Nov. 1998, doi: 10.1109/72.728403.

[44] Y. Jin and B. Sendhoff, "Extracting Interpretable Fuzzy Rules from RBF Networks, ” pp. 149-164, 2003, doi: 10.1023/A:1023642126478.

[45] C. Jia et al., "3D elasticity imaging on an open-chest dog heart," Proc. - IEEE Ultrason. Symp., vol. c, pp. 155-158, 2009, doi: 10.1109/ULTSYM.2009.5441850. 